\title{
Domain-specific and total sedentary behaviors associated with psychological distress in older adults
}

This article was published in the following Dove Press journal: Psychology Research and Behavior Management

\author{
Lovro Štefan' \\ Mario Baić ${ }^{2}$ \\ Goran Sporiš \\ Damir Pekas ${ }^{2}$ \\ Nikola Starčević ${ }^{2}$ \\ 'Faculty of Kinesiology, Department of \\ General and Applied Kinesiology, \\ University of Zagreb, Zagreb, Croatia; \\ ${ }^{2}$ Faculty of Kinesiology, Department of \\ Sport Kinesiology, University of Zagreb, \\ Zagreb, Croatia
}

Purpose: Time spent in sedentary behaviors has become a major public health problem, affecting both physical and mental conditions, which is regularly evident in older adults. The aim of this study was to explore the association between each domain-specific sedentary behavior (screen-time, leisure-time sedentary behavior and transport) and total sedentary behavior (sum of all indicators) with "high" psychological distress among older individuals. Patients and methods: In this cross-sectional study, we recruited 810 participants aged $\geq 85$ (16\% men) from 6 neighborhoods in the city of Zagreb. We used Measure of Older Adults' Sedentary Time sedentary behavior questionnaire to assess the time spent in a specific domain of sedentary behavior and Kessler K6 scale to assess the level of psychological distress. Participants who had a score $\geq 13$ points were treated as those with "high" psychological distress. Generalized estimating equations with Poisson regression models and risk ratios were used to calculate the association.

Results: After adjusting for sex, body mass index, sleep quality, self-rated health, material status, physical activity, diet and chronic diseases, participants categorized in the second, third and fourth quartile of screen-time, in the fourth quartile of leisure-time sedentary behavior and in the third and fourth quartile of total sedentary behavior were less likely to have "high" psychological distress. However, participants categorized in the fourth quartile of transport were more likely to have "high" psychological distress.

Conclusion: Our study shows that more time spent in front of screens, leisure and in total sedentary behavior is associated with lower levels, while more time spent in transport is associated with higher levels of psychological distress, pointing out that the aforementioned associations remained even after adjusting for variables describing "general" physical health. Thus, strategies aiming to reduce the time spent in passive transport and enhance active transport in a sample of older adults are warranted.

Keywords: geriatrics, mental health, sitting, associations, generalized estimating equations

\section{Introduction}

Mental health problems have become a major public health burden. ${ }^{1}$ Along with substance use, they are leading cause of disability worldwide. ${ }^{2}$ In general, mental health can be defined as "a state of well-being in which every individual realizes own potential and can work productively and efficiently to make a contribution to the community". 3 It has been estimated that on average $18 \%$ of the adults experience a common mental disorder within the past 12 months and $29.2 \%$ across lifetime. ${ }^{4}$ Poor mental health increases the risk for developing communicable and
Correspondence: Lovro Štefan

Faculty of Kinesiology, University of

Zagreb, Horvaćanski zavoj 15, Zagreb,

Croatia

Tel +385098917 7060

Email lovro.stefan1510@gmail.com 
non-communicable diseases and injuries leading to premature death. ${ }^{5}$ Psychological distress is one of several mental health issues (depression, anxiety) and is described as "an emotional disturbance that may impact on the social functioning and day-to-day living of individuals". ${ }^{6}$ Higher levels of psychological distress have been consistently associated with several diseases and all-cause mortality. ${ }^{7}$

Psychological distress has many correlates, yet one of them that deserves special investigation are sedentary behaviors. ${ }^{8}$ It is defined as "any waking behavior characterized by an energy expenditure $\leq 1.5$ metabolic equivalents (METs), while in a sitting, reclining or lying posture". 9 Independent of physical activity, sedentary behaviors have often been associated with negative health outcomes, including overweight/obesity status, elevated blood pressure and total cholesterol, lower levels of selfesteem, physical fitness and academic achievement. ${ }^{10}$ Although previous studies have examined the association between sedentary behavior and psychological distress, results have been inconsistent. ${ }^{8,11-13}$ In adults, a study conducted in Scotland showed that more screen-time was not significantly associated with categorically expressed psychological distress. ${ }^{11}$ A study conducted among Australian sample showed that sitting $>6 \mathrm{hrs} /$ day at work/occupation increased the odds of psychological distress. ${ }^{13}$ Among adolescents, studies have shown a strong association between screen-time (a proxy of sedentary behavior) and psychological distress, that is more screen-time is associated with higher levels of psychological distress. ${ }^{14}$ However, studies about various domains of sedentary behavior and psychological distress in older adults ( $\geq 65$ years) from Croatia are lacking.

Although older adults are at decreased risk of mental health problems compared to young adults, those who suffer from poor mental health are at increased risk for having poor health outcomes. ${ }^{15}$ On the other hand, older adults spend more than 4 hrs/day sedentary, while observing domain-specific sedentary behaviors, $65 \%$ of them spend more than $3 \mathrm{hrs} /$ day in front of a screen, over $55 \%$ report watching television for more than $2 \mathrm{hrs} /$ day, ${ }^{16}$ and over $3.3 \mathrm{hrs} /$ day in leisure-time sedentary behavior. ${ }^{17}$ Since the associations between various specific domains of sedentary behavior and psychological distress are still unclear and the majority of the studies used screen-time as a proxy of sedentary behavior, it is necessary to explore and detect a risk group of older adults and create and implement strategies and policies which lower the level of both sedentary time (by substituting with physical activity) and psychological distress. Of the record, regular physical activity is associated with the reduced psychological distress and prevents from falling into a high-risk category of "poor" mental health. ${ }^{18}$ On the other hand, regular physical activity also reduces the likelihood of both psychiatric morbidity and mortality. ${ }^{19}$

Therefore, the main purpose of the present study was to explore the association between each domain-specific sedentary behavior (screen-time, leisure-time sedentary behavior and transport) and total sedentary behavior (sum of all indicators) with "high" psychological distress among older individuals.

\section{Material and methods}

\section{Study participants}

Our sample was based on older adults aged $\geq 85$. A participant selection protocol, inclusion criteria and power analyses are described elsewhere. ${ }^{20}$ In brief, we conveniently chose six neighborhoods from the city of Zagreb. After spreading the information about the main aims, out of 1,040 older adults, our sample eligible for our study was based on 810 elderly individuals. All procedures performed in this study were anonymous and in accordance with the Declaration of Helsinki. The Institutional Review Board of the Faculty of Kinesiology approved the study (Ethics code: 10/7/2018). Before the study, each participant had given their written informed consent to participate in the study.

\section{Psychological distress (outcome variable)}

Psychological distress was assessed using Kessler's sixitem questionnaire: (1) "How often during the past 30 days did you feel nervous?", (2) "How often during the past 30 days did you feel hopeless?", (3) "How often during the past 30 days did you feel restless or fidgety?", (4) "How often during the past 30 days did you feel so depressed that nothing could cheer you up?", (5) "How often during the past 30 days did you feel that everything was an effort?" and (6) "How often during the past 30 days did you feel worthless?". ${ }^{21}$ Each question is scored from 0 (none of the time) to 4 (all the time). The scores of each question are summed up between 0 and 24, with a lower score indicating a lower level of psychological distress. Kessler et $\mathrm{al}^{21}$ showed that responses with $<13$ points vs $\geq 13$ points discriminated participants with and without psychological distress. 


\section{Sedentary behavior assessment (independent variable)}

To assess sedentary behavior, we used Measure of Older Adults' Sedentary Time questionnaire. ${ }^{22}$ In specific, this questionnaire assesses time spent sitting by covering several sedentary behavior domains: (i) watching television, (ii) using a computer/tablet, (ii) reading, (iv) socializing, (v) transport, (vi) hobbies and (vii) total sedentary time. This questionnaire has been shown to have good reliability $(0.52)$ and modest validity $(0.30)$, and it is suitable for use in interventions with older adults. $^{22}$ For the purpose of this study, we created domain-specific categories of sedentary behavior as follows: (1) screen-time (watching television and using a computer/tablet), (2) leisure-time (reading, socializing and hobbies), (3) transport (transport) and (4) total sedentary time (sum of all domain-specific categories). Additionally, we included sex, body mass index, sleep quality, self-rated health, material status, physical activity, diet and chronic diseases as covariates. Briefly, body mass index was self-reported and calculated from height and weight. Sleep quality was measured by using oneitem question: "How would you perceive your sleep quality?" with four possible answers: (1) very poor, (2) poor, (3) good and (4) very good. We categorized the participants into "poor" (very poor and poor) compared to "good" (good and very good) sleep quality. Similarly, self-rated health was assessed by one-item question: 'How would you perceive your health status? on a fivepoint scale: (1) very poor, (2) poor, (3) fair, (4) good and (5) excellent. The result was dichotomized as "poor" (very poor and poor) vs "good" (fair, good and excellent) health. We asked the participants about their material status with two categorizations: (1) below average compared to average/below average. Physical activity in the last seven days was assessed by using International Physical Activity questionnaire, a simple and reliable instrument which provides information about light, moderate and vigorous physical activity. ${ }^{23}$ Participants were categorized as "sufficiently" active (those who participated in at least 150 mins of moderate-intensity aerobic physical activity throughout the week) vs "insufficiently" active (those who did not meet the aforementioned criteria). We used Elderly Diet Index score to assess dietary patterns in older adults. $^{24}$ The questionnaire consists of ten food groups and their frequency intake. Final score ranges between
10 and 40 with higher score indicating higher adherence to dietary recommendations. For the purpose of this study, we categorized the participants as having "low" adherence ( $\leq 28$ points) compared to "moderate/high" adherence ( $>28$ points). The presence of chronic diseases was asked by one question: "Has the doctor ever told you that you suffer from any kind of chronic diseases?" with Yes and No answer.

\section{Data analysis}

Basic descriptive statistics of the participants are presented as frequencies $(\mathrm{N})$ and percentages (\%). First, hours and minutes of sedentary behaviors were transformed to minutes. Next, continuous variable for each domain (screen-time, leisure-time sedentary behavior and transport) and total sedentary time was used to categorize the participants into quartiles. The proportion of the participants in each quartile according to the level of psychological distress ("low" vs "high") was calculated using Chi-square test. Correlation was calculated using Spearman's coefficient of correlation. Next, we used generalized estimating equations with Poisson regression models to calculate the associations between each domain-specific and total sedentary behavior with "high" psychological distress as an outcome variable. We calculated risk ratios (RRs) with $95 \%$ confidence intervals (95\% CIs). In model 1, we examined the association between screen-time and "high" psychological distress. The association between leisure-time sedentary behavior and "high" psychological distress was calculated in Model 2. The association between transport sedentary time and "high" psychological distress was calculated in Model 3. Finally, we examined the association between total sedentary time and "high" psychological distress in Model 4. Each model was adjusted for sex, body mass index, sleep quality, self-rated health, material status, physical activity, diet and chronic diseases. The interaction effect between gender and sedentary behaviors was not statistically significant $(P=0.437)$ and we dropped the gender-stratified analyses. Significance was set up at $\alpha=0.05$. All the analyses were performed in SPSS software, version 22 (IBM, Armonk, NY, USA).

\section{Results}

Basic descriptive statistics of the study participants are presented in Table 1. Higher percentage of the participants who were in the third and fourth quartile of screen-time was categorized in "low" psychological distress group. Also, participants who were categorized in the fourth quartile of leisure-time sedentary behavior and in the third and fourth 
Table I Basic descriptive statistics of the study participants, Croatia (20I8)

\begin{tabular}{|c|c|c|c|c|}
\hline \multirow[t]{2}{*}{ Study variables } & $\begin{array}{l}\text { Total sample } \\
(\mathbf{N}=\mathbf{8} \mid 0)\end{array}$ & $\begin{array}{l}\text { "Low" psychological distress } \\
(\mathrm{N}=610)\end{array}$ & $\begin{array}{l}\text { “High” psychological distress } \\
(\mathrm{N}=200)\end{array}$ & \multirow[t]{2}{*}{$P$-value } \\
\hline & $\mathbf{N}(\%)$ & $\mathbf{N}(\%)$ & $\mathbf{N}(\%)$ & \\
\hline $\begin{array}{l}\text { Screen-time } \\
\text { Quartile I (lowest) } \\
\text { Quartile } 2 \\
\text { Quartile } 3 \\
\text { Quartile } 4 \text { (highest) }\end{array}$ & $\begin{array}{l}249(30.7) \\
167(20.6) \\
204(25.2) \\
190(23.5)\end{array}$ & $\begin{array}{l}169(27.7) \\
119(19.5) \\
168(27.5) \\
154(25.2)\end{array}$ & $\begin{array}{l}80(40.0) \\
48(24.0) \\
36(18.0) \\
36(18.0)\end{array}$ & $<0.001$ \\
\hline $\begin{array}{l}\text { Leisure-time } \\
\text { Quartile I (lowest) } \\
\text { Quartile } 2 \\
\text { Quartile } 3 \\
\text { Quartile } 4 \text { (highest) }\end{array}$ & $\begin{array}{l}247(30.5) \\
144(17.8) \\
216(26.7) \\
203(25.1)\end{array}$ & $\begin{array}{l}169(27.7) \\
105(17.2) \\
157(25.7) \\
179(29.3)\end{array}$ & $\begin{array}{l}78(39.0) \\
39(19.5) \\
59(29.5) \\
24(12.0)\end{array}$ & $<0.00$ I \\
\hline $\begin{array}{l}\text { Transport } \\
\text { Quartile I (lowest) } \\
\text { Quartile } 2 \\
\text { Quartile } 3 \\
\text { Quartile } 4 \text { (highest) }\end{array}$ & $\begin{array}{l}360(44.4) \\
49(5.9) \\
228(28.1) \\
174(21.5)\end{array}$ & $\begin{array}{l}272(44.6) \\
32(5.2) \\
176(28.9) \\
130(21.3)\end{array}$ & $\begin{array}{l}88(44.0) \\
16(8.0) \\
52(26.0) \\
44(22.0)\end{array}$ & 0.492 \\
\hline $\begin{array}{l}\text { Total } \\
\text { Quartile I (lowest) } \\
\text { Quartile } 2 \\
\text { Quartile } 3 \\
\text { Quartile } 4 \text { (highest) }\end{array}$ & $\begin{array}{l}200(24.7) \\
221(27.3) \\
198(24.4) \\
191(23.6)\end{array}$ & $\begin{array}{l}138(22.6) \\
155(25.4) \\
160(26.2) \\
157(25.7)\end{array}$ & $\begin{array}{l}62(31.0) \\
66(33.0) \\
38(19.0) \\
34(17.0)\end{array}$ & $<0.001$ \\
\hline $\begin{array}{l}\text { Sex } \\
\text { Men } \\
\text { Women }\end{array}$ & $\begin{array}{l}132(16.3) \\
678(83.7)\end{array}$ & $\begin{array}{l}84(13.8) \\
526(86.2)\end{array}$ & $\begin{array}{l}48(24.0) \\
152(76.0)\end{array}$ & $<0.001$ \\
\hline $\begin{array}{l}\text { Body mass index } \\
\text { Normal } \\
\text { Overweight/obese }\end{array}$ & $\begin{array}{l}298(36.8) \\
512(63.2)\end{array}$ & $\begin{array}{l}234(38.4) \\
376(61.6)\end{array}$ & $\begin{array}{l}64(32.0) \\
136(68.0)\end{array}$ & 0.109 \\
\hline $\begin{array}{l}\text { Sleep quality } \\
\text { Good } \\
\text { Poor }\end{array}$ & $\begin{array}{l}302(37.3) \\
508(62.7)\end{array}$ & $\begin{array}{l}188(30.8) \\
422(69.2)\end{array}$ & $\begin{array}{l}114(57.0) \\
86(43.0)\end{array}$ & $<0.001$ \\
\hline $\begin{array}{l}\text { Self-rated health } \\
\text { Good } \\
\text { Poor }\end{array}$ & $\begin{array}{l}396(48.8) \\
4 I 4(5 I .2)\end{array}$ & $\begin{array}{l}336(54.9) \\
274(45.1)\end{array}$ & $\begin{array}{l}60(30.0) \\
140(70.0)\end{array}$ & $<0.001$ \\
\hline $\begin{array}{l}\text { Material status } \\
\text { Middle-high } \\
\text { Low }\end{array}$ & $\begin{array}{l}132(16.3) \\
678(83.7)\end{array}$ & $\begin{array}{l}514(84.3) \\
96(15.7)\end{array}$ & $\begin{array}{l}164(82.0) \\
36(18.0)\end{array}$ & 0.442 \\
\hline $\begin{array}{l}\text { Physical activity } \\
\text { Sufficient } \\
\text { Insufficient }\end{array}$ & $\begin{array}{l}180(22.2) \\
630(77.8)\end{array}$ & $\begin{array}{l}153(25.1) \\
457(74.9)\end{array}$ & $\begin{array}{l}27(13.5) \\
173(86.5)\end{array}$ & $<0.001$ \\
\hline $\begin{array}{l}\text { Elderly Diet Index } \\
\text { Moderate-high } \\
\text { Low }\end{array}$ & $\begin{array}{l}144(17.8) \\
666(82.2)\end{array}$ & $\begin{array}{l}130(21.3) \\
480(78.7)\end{array}$ & $\begin{array}{l}14(7.0) \\
186(93.0)\end{array}$ & $<0.001$ \\
\hline $\begin{array}{l}\text { Chronic diseases } \\
\text { No } \\
\text { Yes }\end{array}$ & $\begin{array}{l}280(34.6) \\
530(65.4)\end{array}$ & $\begin{array}{l}212(34.8) \\
398(65.2)\end{array}$ & $\begin{array}{l}68(34.0) \\
132(66.0)\end{array}$ & 0.864 \\
\hline
\end{tabular}


quartile of total sedentary behavior were more likely to be categorized as those with "low" psychological distress. On the other hand, higher percentage of the participants who were in the fourth quartile of transport was more likely to be categorized in "high" psychological distress group. Also, those who were overweight/obese, "poor" sleepers, reporting "poor" self-rated health, those who were "insufficiently" active and who had "low" adherence to diet were more likely to be categorized in "high" psychological distress group.

Table 2 shows correlations between the study variables. In general, Spearman's correlation showed that the majority of the study variables were significantly associated to each other. Specifically, leisure-time sedentary behavior $(r=-0.16)$ and screen-time sedentary behavior $(r=-0.14)$ had stronger association with psychological distress, compared to transport. Also, both leisure-time sedentary behavior $(r=0.81)$ and screen-time $(r=0.66)$ were strongly associated with total sedentary behavior, compared to transport $(r=0.27)$

Table 3 shows non-adjusted associations between domain-specific (screen-time, leisure-time sedentary behavior and transport) and total sedentary time and "high" psychological distress. In Model 1, participants categorized in the second $(\mathrm{RR}=0.97 ; 9 \%$ CI 0.96-0.99), third $(\mathrm{RR}=0.89 ; 95 \%$ CI $0.89-0.89)$ and fourth $(\mathrm{RR}=0.90$; 95\% CI 0.84-0.96) quartile of screen-time were less likely to report "high" psychological distress. In Model 2, participants categorized in the highest quartile of leisure-time sedentary behavior $(\mathrm{RR}=0.85$; 95\% CI $0.84-0.86)$ were also less likely to report "high" psychological distress and in Model 4, participants who were in the third $(\mathrm{RR}=0.91 ; 95 \%$ CI $0.91-0.91)$ and fourth $(\mathrm{RR}=0.90$; $95 \%$ CI $0.77-1.00)$ quartile of total sedentary behavior were less likely to report "high" psychological distress. The association between transport and "high" psychological distress was not statistically significant.

Table 4 shows adjusted associations between domainspecific (screen-time, leisure-time sedentary behavior and transport) and total sedentary time and "high" psychological distress. In Model 1, participants categorized in the second $(\mathrm{RR}=0.95 ; 9 \%$ CI $0.90-1.00)$, third $(\mathrm{RR}=0.92 ; 95 \% \mathrm{CI}$ $0.90-0.95)$ and fourth $(\mathrm{RR}=0.92 ; 95 \%$ CI $0.89-0.95)$ quartile of screen-time were less likely to report "high" psychological distress. In Model 2, participants categorized in the highest quartile of leisure-time sedentary behavior $(\mathrm{RR}=0.88$; $95 \% \mathrm{CI}$ 0.87-0.89) were also less likely to report "high" psychological distress and in Model 4, participants who were in the third $(\mathrm{RR}=0.93 ; 95 \% \mathrm{CI} 0.93-0.93)$ and fourth $(\mathrm{RR}=0.92 ; 95 \% \mathrm{CI}$ 0.85-0.99) quartile of total sedentary behavior were less likely to report "high" psychological distress. However, categorized in the fourth $(\mathrm{RR}=1.08 ; 95 \% \mathrm{CI} 1.05-1.11)$ quartile of transport were more likely to report "high" psychological distress.

\section{Discussion}

The main purpose of the present study was to explore the association between each domain-specific sedentary behavior (screen-time, leisure-time and transport) and total sedentary behavior with "high" psychological distress among older individuals. This is the first study examining the aforementioned associations in older adults.

First, our study showed that more time spent in front of screens (television and computer/tablet) was associated with lower levels of psychological distress. Our findings are not in line with previous studies exploring the same associations in adult populations. ${ }^{8,11-13}$ Such findings

Table 2 Correlation analysis between the study variables

\begin{tabular}{|c|c|c|c|c|c|c|c|c|c|c|c|c|c|}
\hline Study variables & I & 2 & 3 & 4 & 5 & 6 & 7 & 8 & 9 & 10 & I I & 12 & 13 \\
\hline Psychological distress (I) & 1 & & & & & & & & & & & & \\
\hline Screen-time (2) & $-0.14^{\mathrm{c}}$ & I & & & & & & & & & & & \\
\hline Leisure-time (3) & $-0.16^{c}$ & $0.4 I^{\mathrm{c}}$ & I & & & & & & & & & & \\
\hline Transport (4) & -0.01 & 0.01 & 0.05 & I & & & & & & & & & \\
\hline Total (5) & $-0.13^{c}$ & $0.66^{\mathrm{c}}$ & $0.8 I^{\mathrm{c}}$ & $0.27^{c}$ & I & & & & & & & & \\
\hline $\operatorname{Sex}(6)$ & $-0.12^{c}$ & 0.02 & $0.15^{c}$ & 0.02 & $0.08^{\mathrm{a}}$ & I & & & & & & & \\
\hline Body mass index (7) & 0.06 & -0.01 & 0.01 & $-0.07^{\mathrm{a}}$ & 0.03 & $-0.16^{\mathrm{c}}$ & I & & & & & & \\
\hline Sleep quality (8) & $-0.23^{c}$ & $0.08^{\mathrm{a}}$ & 0.04 & $0.21^{\mathrm{c}}$ & $0.07^{\mathrm{a}}$ & 0.06 & 0.01 & I & & & & & \\
\hline Self-rated health (9) & $-0.21^{c}$ & 0.02 & -0.02 & 0.05 & $-0.08^{\mathrm{a}}$ & 0.01 & -0.04 & $0.32^{c}$ & I & & & & \\
\hline Material status $(10)$ & -0.03 & $0.11^{\mathrm{b}}$ & $0.08^{\mathrm{a}}$ & 0.06 & $0.10^{\mathrm{b}}$ & $-0.09^{b}$ & -0.01 & $0.12^{c}$ & $0.16^{\mathrm{c}}$ & I & & & \\
\hline Physical activity (II) & $-0.12^{c}$ & $0.16^{\mathrm{c}}$ & $0.15^{c}$ & $0.10^{\mathrm{b}}$ & $0.14^{\mathrm{c}}$ & $0.13^{c}$ & -0.05 & $0.14^{c}$ & $0.13^{c}$ & $-0.18^{c}$ & I & & \\
\hline Elderly Diet Index (12) & $-0.16^{c}$ & $0.08^{\mathrm{a}}$ & 0.06 & $0.17^{c}$ & $0.08^{\mathrm{a}}$ & -0.02 & $-0.15^{\mathrm{c}}$ & $-0.07^{\mathrm{a}}$ & $0.15^{c}$ & $-0.07^{\mathrm{a}}$ & $0.24^{c}$ & $\mathrm{I}$ & \\
\hline Chronic diseases (I3) & -0.01 & $-0.11^{b}$ & $-0.12^{c}$ & $0.08^{\mathrm{a}}$ & $-0.11^{b}$ & $-0.11^{b}$ & $-0.17^{c}$ & $0.16^{c}$ & $0.22^{\mathrm{c}}$ & $0.08^{\mathrm{a}}$ & -0.04 & 0.03 & I \\
\hline
\end{tabular}

Note: ${ }^{a} P<0.05 ;{ }^{b} p<0.01 ;{ }^{c} P<0.001$. 
Table 3 Non-adjusted estimates between domain-specific (screen-time, leisure-time and transport) and total sedentary behavior with "high" psychological distress in the study participants, Croatia (2018)

\begin{tabular}{|c|c|c|c|c|}
\hline \multirow[t]{2}{*}{ Study variables } & Model I & Model 2 & Model 3 & Model 4 \\
\hline & $\operatorname{RR}(95 \% \mathrm{CI})$ & $\operatorname{RR}(95 \% \mathrm{CI})$ & RR (95\% CI) & RR (95\% CI) \\
\hline $\begin{array}{l}\text { Screen-time } \\
\text { Quartile I (lowest) } \\
\text { Quartile } 2 \\
\text { Quartile } 3 \\
\text { Quartile } 4 \text { (highest) }\end{array}$ & $\begin{array}{l}\text { Ref. } \\
0.97(0.96-0.99)^{\mathrm{a}} \\
0.89(0.89-0.89)^{\mathrm{c}} \\
0.90(0.84-0.96)^{\mathrm{b}}\end{array}$ & & & \\
\hline $\begin{array}{l}\text { Leisure-time } \\
\text { Quartile I (lowest) } \\
\text { Quartile } 2 \\
\text { Quartile } 3 \\
\text { Quartile } 4 \text { (highest) }\end{array}$ & & $\begin{array}{l}\text { Ref. } \\
0.97(0.82-1.14) \\
0.97(0.82-1.06) \\
0.85(0.84-0.86)^{\mathrm{c}}\end{array}$ & & \\
\hline $\begin{array}{l}\text { Transport } \\
\text { Quartile I (lowest) } \\
\text { Quartile } 2 \\
\text { Quartile } 3 \\
\text { Quartile } 4 \text { (highest) }\end{array}$ & & & $\begin{array}{l}\text { Ref. } \\
1.07(0.92-1.25) \\
0.99(0.95-1.02) \\
1.00(0.98-1.02)\end{array}$ & \\
\hline $\begin{array}{l}\text { Total } \\
\text { Quartile I (lowest) } \\
\text { Quartile } 2 \\
\text { Quartile } 3 \\
\text { Quartile } 4 \text { (highest) }\end{array}$ & & & & $\begin{array}{l}\text { Ref. } \\
0.99(0.88-1.12) \\
0.91(0.91-0.91)^{c} \\
0.90(0.77-1.00)^{2}\end{array}$ \\
\hline $\begin{array}{l}\text { Sex } \\
\text { Men } \\
\text { Women }\end{array}$ & $\begin{array}{l}\text { Ref. } \\
0.90(0.90-0.90)^{c}\end{array}$ & $\begin{array}{l}\text { Ref. } \\
0.90(0.90-0.90)^{c}\end{array}$ & $\begin{array}{l}\text { Ref. } \\
0.90(0.90-0.90)^{c}\end{array}$ & $\begin{array}{l}\text { Ref. } \\
0.90(0.90-0.90)^{c}\end{array}$ \\
\hline $\begin{array}{l}\text { Body mass index } \\
\text { Normal } \\
\text { Overweight/obese }\end{array}$ & $\begin{array}{l}\text { Ref. } \\
\text { I.04 }(1.01-1.08)^{\mathrm{a}}\end{array}$ & $\begin{array}{l}\text { Ref. } \\
1.04(1.01-1.08)^{\mathrm{a}}\end{array}$ & $\begin{array}{l}\text { Ref. } \\
1.04(1.01-1.08)^{\mathrm{a}}\end{array}$ & $\begin{array}{l}\text { Ref. } \\
1.04(1.01-1.08)^{2}\end{array}$ \\
\hline $\begin{array}{l}\text { Sleep quality } \\
\text { Good } \\
\text { Poor }\end{array}$ & $\begin{array}{l}\text { Ref. } \\
\text { I.I8 (1.17-I.18) }\end{array}$ & $\begin{array}{l}\text { Ref. } \\
1.18(1.17-1.18)^{\mathrm{c}}\end{array}$ & $\begin{array}{l}\text { Ref. } \\
1.18(1.17-1.18)^{c}\end{array}$ & $\begin{array}{l}\text { Ref. } \\
\text { I.18 (1.17-I.18) }\end{array}$ \\
\hline $\begin{array}{l}\text { Self-rated health } \\
\text { Good } \\
\text { Poor }\end{array}$ & $\begin{array}{l}\text { Ref. } \\
1.16(1.08-1.25)^{c}\end{array}$ & $\begin{array}{l}\text { Ref. } \\
1.16(1.08-1.25)^{\mathrm{c}}\end{array}$ & $\begin{array}{l}\text { Ref. } \\
1.16(1.08-1.25)^{c}\end{array}$ & $\begin{array}{l}\text { Ref. } \\
1.16(1.08-1.25)^{c}\end{array}$ \\
\hline $\begin{array}{l}\text { Material status } \\
\text { Middle-high } \\
\text { Low }\end{array}$ & $\begin{array}{l}\text { Ref. } \\
1.02(0.99-1.06)\end{array}$ & $\begin{array}{l}\text { Ref. } \\
1.02(0.99-1.06)\end{array}$ & $\begin{array}{l}\text { Ref. } \\
1.02(0.99-1.06)\end{array}$ & $\begin{array}{l}\text { Ref. } \\
1.02(0.99-1.06)\end{array}$ \\
\hline $\begin{array}{l}\text { Physical activity } \\
\text { Sufficient } \\
\text { Insufficient }\end{array}$ & $\begin{array}{l}\text { Ref. } \\
\text { I.II }(1.08-1.13)^{c}\end{array}$ & $\begin{array}{l}\text { Ref. } \\
1.11(1.08-1.13)^{\mathrm{c}}\end{array}$ & $\begin{array}{l}\text { Ref. } \\
\text { I.II }(1.08-1.13)^{c}\end{array}$ & $\begin{array}{l}\text { Ref. } \\
\text { I.II }(1.08-1.13)^{c}\end{array}$ \\
\hline $\begin{array}{l}\text { Elderly Diet Index } \\
\text { Moderate-high } \\
\text { Low }\end{array}$ & $\begin{array}{l}\text { Ref. } \\
1.17(1.14-1.19)^{c}\end{array}$ & $\begin{array}{l}\text { Ref. } \\
1.17(1.14-1.19)^{\mathrm{c}}\end{array}$ & $\begin{array}{l}\text { Ref. } \\
1.17(1.14-1.19)^{c}\end{array}$ & $\begin{array}{l}\text { Ref. } \\
1.17(1.14-1.19)\end{array}$ \\
\hline $\begin{array}{l}\text { Chronic diseases } \\
\text { No } \\
\text { Yes }\end{array}$ & $\begin{array}{l}\text { Ref. } \\
0.99(0.94-1.05)\end{array}$ & $\begin{array}{l}\text { Ref. } \\
0.99(0.94-1.05)\end{array}$ & $\begin{array}{l}\text { Ref. } \\
0.99(0.94-1.05)\end{array}$ & $\begin{array}{l}\text { Ref. } \\
0.99(0.94-1.05)\end{array}$ \\
\hline
\end{tabular}

Notes: Each variable was put separately into the models. ${ }^{a} P<0.05$; ${ }^{b} P<0.01 ;{ }^{c} P<0.001$. 
Table 4 Adjusted estimates between domain-specific (screen-time, leisure-time and transport) and total sedentary behavior with "high" psychological distress in the study participants, Croatia (2018)

\begin{tabular}{|c|c|c|c|c|}
\hline \multirow[t]{2}{*}{ Study variables } & Model I & Model 2 & Model 3 & Model 4 \\
\hline & RR (95\% Cl) & $\operatorname{RR}(95 \% \mathrm{CI})$ & RR (95\% CI) & RR (95\% Cl) \\
\hline $\begin{array}{l}\text { Screen-time } \\
\text { Quartile I (lowest) } \\
\text { Quartile } 2 \\
\text { Quartile } 3 \\
\text { Quartile } 4 \text { (highest) }\end{array}$ & $\begin{array}{l}\text { Ref. } \\
0.95(0.90-1.00)^{\mathrm{a}} \\
0.92(0.90-0.95)^{\mathrm{c}} \\
0.92(0.89-0.95)^{\mathrm{c}}\end{array}$ & & & \\
\hline $\begin{array}{l}\text { Leisure-time } \\
\text { Quartile I (lowest) } \\
\text { Quartile } 2 \\
\text { Quartile } 3 \\
\text { Quartile } 4 \text { (highest) }\end{array}$ & & $\begin{array}{l}\text { Ref. } \\
0.98(0.83-1.15) \\
0.97(0.90-1.05) \\
0.88(0.87-0.89)^{\mathrm{c}}\end{array}$ & & \\
\hline $\begin{array}{l}\text { Transport } \\
\text { Quartile I (lowest) } \\
\text { Quartile } 2 \\
\text { Quartile } 3 \\
\text { Quartile } 4 \text { (highest) }\end{array}$ & & & $\begin{array}{l}\text { Ref. } \\
\text { I.02 }(0.92-1.13) \\
1.02(0.99-1.06) \\
1.08(1.05-1.11)^{c}\end{array}$ & \\
\hline $\begin{array}{l}\text { Total } \\
\text { Quartile I (lowest) } \\
\text { Quartile } 2 \\
\text { Quartile } 3 \\
\text { Quartile } 4 \text { (highest) }\end{array}$ & & & & $\begin{array}{l}\text { Ref. } \\
\text { I.00 }(0.94-1.07) \\
0.93(0.93-0.93)^{\mathrm{c}} \\
0.92(0.85-0.99)^{\mathrm{a}}\end{array}$ \\
\hline $\begin{array}{l}\text { Sex } \\
\text { Men } \\
\text { Women }\end{array}$ & $\begin{array}{l}\text { Ref. } \\
0.92(0.91-0.93)^{c}\end{array}$ & $\begin{array}{l}\text { Ref. } \\
0.93(0.90-0.95)^{c}\end{array}$ & $\begin{array}{l}\text { Ref. } \\
0.91(0.91-0.92)^{c}\end{array}$ & $\begin{array}{l}\text { Ref. } \\
0.92(0.92-0.93)^{c}\end{array}$ \\
\hline $\begin{array}{l}\text { Body mass index } \\
\text { Normal } \\
\text { Overweight/obese }\end{array}$ & $\begin{array}{l}\text { Ref. } \\
1.01(1.01-1.02)^{c}\end{array}$ & $\begin{array}{l}\text { Ref. } \\
1.02(1.02-1.03)^{c}\end{array}$ & $\begin{array}{l}\text { Ref. } \\
1.02(1.01-1.03)^{c}\end{array}$ & $\begin{array}{l}\text { Ref. } \\
1.02(1.01-1.03)^{c}\end{array}$ \\
\hline $\begin{array}{l}\text { Sleep quality } \\
\text { Good } \\
\text { Poor }\end{array}$ & $\begin{array}{l}\text { Ref. } \\
1.14(1.12-1.16)^{c}\end{array}$ & $\begin{array}{l}\text { Ref. } \\
1.14(1.11-1.17)^{c}\end{array}$ & $\begin{array}{l}\text { Ref. } \\
1.16(1.14-1.18)^{c}\end{array}$ & $\begin{array}{l}\text { Ref. } \\
\text { I.I4 (1.14-I.15) }\end{array}$ \\
\hline $\begin{array}{l}\text { Self-rated health } \\
\text { Good } \\
\text { Poor }\end{array}$ & $\begin{array}{l}\text { Ref. } \\
1.10(1.02-1.19)^{\mathrm{b}}\end{array}$ & $\begin{array}{l}\text { Ref. } \\
1.10(1.03-1.18)^{\mathrm{b}}\end{array}$ & $\begin{array}{l}\text { Ref. } \\
1.09(1.03-1.16)^{\mathrm{b}}\end{array}$ & $\begin{array}{l}\text { Ref. } \\
\text { I.II }(1.04-1.18)^{b}\end{array}$ \\
\hline $\begin{array}{l}\text { Material status } \\
\text { Middle-high } \\
\text { Low }\end{array}$ & $\begin{array}{l}\text { Ref. } \\
1.00(0.96-1.06)\end{array}$ & $\begin{array}{l}\text { Ref. } \\
1.00(0.96-10.6)\end{array}$ & $\begin{array}{l}\text { Ref. } \\
\text { I.0I }(0.95-1.08)\end{array}$ & $\begin{array}{l}\text { Ref. } \\
1.00(0.94-1.07)\end{array}$ \\
\hline $\begin{array}{l}\text { Physical activity } \\
\text { Sufficient } \\
\text { Insufficient }\end{array}$ & $\begin{array}{l}\text { Ref. } \\
\text { I.0I (0.95-I.07) }\end{array}$ & $\begin{array}{l}\text { Ref. } \\
\text { I.0I }(0.96-1.05)\end{array}$ & $\begin{array}{l}\text { Ref. } \\
1.03(0.97-1.09)\end{array}$ & $\begin{array}{l}\text { Ref. } \\
1.01(0.96-1.07)\end{array}$ \\
\hline $\begin{array}{l}\text { Elderly Diet Index } \\
\text { Moderate-high } \\
\text { Low }\end{array}$ & $\begin{array}{l}\text { Ref. } \\
1.15(1.15-1.15)^{c}\end{array}$ & $\begin{array}{l}\text { Ref. } \\
1.14(1.12-1.16)^{c}\end{array}$ & $\begin{array}{l}\text { Ref. } \\
1.17(1.13-1.20)^{c}\end{array}$ & 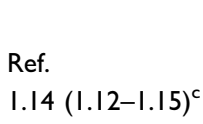 \\
\hline $\begin{array}{l}\text { Chronic diseases } \\
\text { No } \\
\text { Yes }\end{array}$ & $\begin{array}{l}\text { Ref. } \\
\text { I.03 }(0.96-1.09)\end{array}$ & $\begin{array}{l}\text { Ref. } \\
1.03(0.96-1.09)\end{array}$ & $\begin{array}{l}\text { Ref. } \\
1.03(0.98-1.09)\end{array}$ & $\begin{array}{l}\text { Ref. } \\
1.03(0.98-1.09)\end{array}$ \\
\hline
\end{tabular}

Notes: Model I examines the association between screen-time and "high" psychological distress adjusted for sex, body mass index, sleep quality, self-rated health, material status, physical activity, diet and chronic diseases. Model 2 examines the association between leisure-time and "high" psychological distress adjusted for sex, body mass index, sleep quality, self-rated health, material status, physical activity, diet and chronic diseases. Model $\mathbf{3}$ examines the association between transport and "high" psychological distress adjusted for sex, body mass index, sleep quality, self-rated health, material status, physical activity, diet and chronic diseases. Model 4 examines the association between total sedentary behavior and "high" psychological distress adjusted for sex, body mass index, sleep quality, self-rated health, material status, physical activity, diet and chronic diseases. ${ }^{\mathrm{a}} \mathrm{P}<0.05$; ${ }^{\mathrm{b}} \mathrm{P}<0.0 \mathrm{I} ;{ }^{\mathrm{c}} \mathrm{P}<0.00 \mathrm{I}$. 
could be explained by several mechanisms. First, the majority of previous studies have been conducted among adolescent ${ }^{14}$ and adult ${ }^{8,11,13}$ populations who are at more extreme risk of having some kind of mental disorder, compared to older adults. ${ }^{15}$ Second, one previous study exploring what older adults think of television in the context of depression showed that participants with low education mentioned that television could be helpful in decreasing depression symptoms. ${ }^{25}$ Since our results showed that watching television and being on the computer/tablet lower the level of psychological distress, such association could be possibly driven by a high percentage of our participants $(83.7 \%)$ who reported having low material status (which is remotely associated with educational level). Third, older adults in general do not seem to experience the same stress-buffering effects watching television compared to young and middle-aged adults. ${ }^{26}$ They also report more positive affects, less negative affects and greater life satisfaction by being in front of screens. ${ }^{27}$ Finally, one previous study showed that the attenuated effect of the time watching television and experienced stress was mediated by lower stress ratings among older adults, which is not surprising since a significant smaller percent of participants reported experiencing "high" psychological distress in the last month. ${ }^{26}$

Next, our results also showed that more sedentary time spent in leisure was associated with lower likelihood of having "high" psychological distress. As in one previous study, ${ }^{28}$ leisure-time sedentary behavior was the most frequent purpose of sedentary behavior in our study. It is worthwhile of noticing that we summed the time spent reading, hanging out with family and friends/socializing and doing hobbies. Thus, most of these behaviors are done in groups and previous studies have questioned whether social time spent in aforementioned behaviors could possibly be good for health in older adults, even though it is predominantly sedentary. ${ }^{29}$ One study showed that depression can be influenced by social isolation (loneliness) leading to the conclusion that social activities, especially done in groups could have beneficial effects on mental health. ${ }^{29}$ Another study showed that cognitively demanding activities, like reading or solving crosswords, may even facilitate cognitive functioning. ${ }^{30}$ Finally, $7 \%$ of our participants reported helping others by taking care of them, increasing social interaction and a sense of a societal role. ${ }^{31}$
Our findings suggest that older adults who spent the highest amount of time in transport were more likely to have "high" psychological distress. Our results are in line with the previous one. ${ }^{32}$ Specifically, Office for National Statistics showed a strong negative association between passive commuting and well-being, that is commuting by bus or rail for more than 30 mins increase the odds of "poor" wellbeing, in comparison to shorter journeys by any mode. The same report stated that possible reasons for such association might be short-term constraints, changes in circumstance and not willing to change commuting patterns where individuals might not recognize potential benefits of changing commute (for example, from passive to active) on both physical and mental health. ${ }^{32}$

Finally, our results showed that participants who were in the highest quartile of total sedentary time were at decreased risk of being highly distressed, compared to those who were in the first quartile. As for screen- and leisure-time sedentary behaviors, our results are contrary to other findings on this topic. ${ }^{8,11-13}$ Leisure-time and screen-time sedentary behaviors had stronger association with total sedentary behavior, compared to transport. Therefore, it is possible that the association between total sedentary behavior and psychological distress in our study was driven by domain-specific and separate associations of screen-time and leisure-time with psychological distress. Since screen- and leisure-time sedentary behaviors are often done in groups (socializing, watching television and doing hobbies together), it seems that they have beneficial effects on mental health in older adults, although being sedentary.

This study has a few strengths. First, we based our study on a relatively large sample of older adults. Second, we used numerous covariates to adjust for potential associations between domain-specific sedentary behaviors and total sedentary behavior and "high" psychological distress. In addition, we adjusted for body mass index. Recently, a study by Hamer and Stamatakis ${ }^{33}$ showed a U-shaped association between body mass index and psychological distress. On the other hand, sedentary behavior interventions, although with small clinical effect must be incorporated in multicomponent interventions for treating obese individuals. $^{34}$

Our study has several limitations. First, by using a cross-sectional design of the study, we cannot define the causality of the association, so our findings must be interpreted with caution. Second, we used subjective 
measures to assess domain-specific sedentary behaviors and psychological distress. One previous study showed that self-reported sedentary time was $3.6 \mathrm{hrs} /$ day lower than accelerometer-derived sedentary time, so it is possible that participants in our study under-reported their domainspecific sedentary time, which might have led to potential measurement error. ${ }^{22}$ Third, we conveniently chose six neighborhoods from the city of Zagreb, limiting the generalizability and practical implications of our results to other older individuals who are in nursing homes or situated in other neighborhoods. Fourth, we additionally adjusted for sleep quality and since psychological distress includes difficulty in sleeping, it is possible that by including sleep quality as a potential covariate, estimates are likely to be under-estimated. Finally, we did not include genetic factors (specific genes) as potential covariates, since one previous study has shown that genetic features might be associated with the occurrence of "poor" mental health. ${ }^{35}$ Future studies should use objective measures (accelerometers) and detailed check-ups by medical doctors in psychology/psychiatry institutions in a follow-up design (longitudinal), in order to establish causal association between domain-specific and total sedentary behaviors and psychological distress in older adults.

\section{Conclusion}

Our study shows that more time spent in front of screens, leisure and in total sedentary behavior is associated with lower levels, while more time spent in transport is associated with higher levels of psychological distress. Thus, by implementing strategies that would increase active transport and leverage social capital throughout bonding and socializing in communities of older adults are warranted.

\section{Ethical approval}

This study received ethical approval from the Institutional Review Board of the Faculty of Kinesiology, University of Zagreb, Croatia. Written informed consent was obtained with all patients participating in this study.

\section{Data sharing statement}

All the data can be accessed by reasonable request to the corresponding author.

\section{Acknowledgments}

We would like to thank all the participants for their enthusiastic participation in the study. This paper was self-funded.

\section{Author contributions}

All authors contributed to data analysis, drafting and revising the article, gave final approval of the version to be published, and agree to be accountable for all aspects of the work

\section{Disclosure}

The authors report no conflicts of interest in this work.

\section{References}

1. Wainberg ML, Scorza P, Shultz JM, et al. Challenges and opportunities in global mental health: a research-to-practice perspective. Curr Psychiatry Rep. 2017;19:28. doi:10.1007/ s11920-017-0780-z

2. Whiteford HA, Degenhardt L, Rehm J, et al. Global burden of disease attributable to mental and substance use disorders: findings from the global burden of disease study 2010. Lancet. 2013;382:1575. doi:10.1016/S0140-6736(13)61611-6

3. Internet page. Available from: http://www.who.int/features/factfiles/ mental_health/en/. Accessed November 30, 2018.

4. Steel Z, Marnane C, Iranpour C, et al. The global prevalence of common mental disorders: a systematic review and meta-analysis 1980-2013. Int $J$ Epidemiol. 2014;43:476-493. doi:10.1093/ije/ dyu038

5. McLachlan KJJ, Gale CR. The effects of psychological distress and its interaction with socioeconomic position on risk of developing four chronic diseases. J Psychosom Res. 2018;109:79-85. doi:10.1016/j. jpsychores.2018.04.004

6. Wheaton B. The twain meet: distress, disorder and the continuing conundrum of categories (comment on Horwitz). Health (London). 2007;11:303-319. doi:10.1177/1363459307077545

7. Russ TC, Stamatakis E, Hamer M, et al. Association between psychological distress and mortality: individual participant pooled analysis of 10 prospective cohort studies. BMJ. 2012;345:e4933. doi:10.1136/bmj.e4933

8. Sloan RA, Sawada SS, Girdano D, et al. Associations of sedentary behavior and physical activity with psychological distress: a cross-sectional study from Singapore. BMC Public Health. 2013;13:885. doi:10.1186/1471-2458-13-885

9. Tremblay MS, Aubert S, Barnes JD, et al. Sedentary Behavior Research Network (SBRN) - terminology consensus project process and outcome. Int J Behav Nutr Phys Act. 2017;14(1):75. doi:10.1186/ s12966-017-0525-8

10. De Rezende LFM, Rodrigues Lopes M, Rey-López JP, et al. Sedentary behavior and health outcomes: an overview of systematic reviews. PLoS One. 2014;9:e105620. doi:10.1371/journal. pone. 0105620

11. Hamer M, Stamatakis E, Mishra GD. Television- and screen-based activity and mental well-being in adults. Am J Prev Med. 2010;38:375-380. doi:10.1016/j.amepre. 2009.12.030

12. Atkin AJ, Adams E, Bull FC, Biddle SJH. Non-occupational sitting and mental well-being in employed adults. Ann Behav Med. 2012;43:181-188. doi:10.1007/s12160-011-9320-y

13. Kilpatrick M, Sanderson K, Blizzard L, et al. Cross-sectional associations between sitting at work and psychological distress: reducing sitting time may benefit mental health. Ment Health Physic Activ. 2013;6:103-109. doi:10.1016/j.mhpa.2013.06.004

14. Hoare E, Milton K, Foster C, et al. The associations between sedentary behaviour and mental health among adolescents: a systematic review. Int J Behav Nutr Phys Act. 2016;13:108. doi:10.1186/s12966-016-0432-4 
15. Fiske A, Wetherell JL, Gatz M. Depression in older adults. Annu Rev Clin Psychol. 2009;5:363-389. doi:10.1146/annurev.clinpsy.032408.153621

16. Harvey JA, Chastin SF, Skelton DA. Prevalence of sedentary behavior in older adults: a systematic review. Int $J$ Environ Res Public Health. 2013;10(12):6645-6661. doi:10.3390/ijerph10126645

17. Harvey JA, Chastin SF, Skelton DA. How sedentary are older people? A systematic review of the amount of sedentary behavior. J Aging Phys Act. 2015;23:471-487. doi:10.1123/japa.2014-0164

18. Perales F, Del Pozo-Cruz J, Del Pozo-Cruz B. Impact of physical activity on psychological distress: a prospective analysis of an Australian national sample. Am $J$ Public Health. 2014;104:91-97. doi:10.2105/ AJPH.2014.302169

19. Chekroud SR, Gueorguieva R, Zheutlin AB, et al. Association between physical exercise and mental health in 1.2 million individuals in the USA between 2011 and 2015: a cross-sectional study. Lancet. 2018;5:739-746. doi:10.1016/S2215-0366(18)30227-X

20. Štefan L, Radman I, Podnar H, Vrgoč G. Sleep duration and sleep quality associated with dietary index in free-living very old adults. Nutrients. 2018;10:e1748. doi:10.3390/nu10111748

21. Kessler RC, Barker PR, Colpe LJ, et al. Screening for serious mental illness in the general population. Arc Gen Psychiatry. 2003;60:184-189. doi:10.1001/archpsyc.60.2.184

22. Gardiner PA, Clark BK, Healy GN, et al. Measuring older adults' sedentary time: reliability, validity, and responsiveness. Med Sci Sports Exerc. 2011;43:2127-2133. doi:10.1249/ MSS.0b013e31821b94f7

23. Hurtig-Wehnlöf A, Hagströmer M, Olsson LA. The international physical activity questionnaire modified for the elderly: aspects of validity and feasibility. Public Health Nutr. 2010;13:1847-1854. doi:10.1017/S1368980009992163

24. Kourlaba G, Polychronopoulos E, Zampelas A, et al. Development of a diet index for older adults and its relation to cardiovascular disease risk factors: the Elderly Dietary Index. $J$ Am Diet Assoc. 2009;109:1022-1030. doi:10.1016/j.jada.2009.03.004
25. Nguyen GT, Wittink MN, Murray GF, et al. More than just a communication medium: what older adults say about television and depression. Gerontologist. 2008;48:300-310.

26. Depp CA, Schkade DA, Thompson WK, Jeste DV. Age, affective experience, and television use. Am J Prev Med. 2010;39:173-178. doi:10.1016/j.amepre.2010.03.020

27. Mroczek DKIII. Change in life satisfaction during adulthood: findings from the veterans affairs normative aging study. J Pers Soc Psychol. 2005;88:189-202. doi:10.1037/0022-3514.88.3.421

28. Leask CF, Harvey JA, Skelton DA, et al. Exploring the context of sedentary behaviour in older adults (what, where, why, when and with whom). Eur Rev Aging Phys Act. 2015;12:4. doi:10.1186/s11556-015-0146-7

29. Alpass FM, Neville S. Loneliness, health and depression in older males. Aging Ment Health. 2003;7:212-216. doi:10.1080/ 1360786031000101193

30. Stamatakis E, Davis M, Stathi A, et al. Associations between multiple indicators of objectively-measured and self-reported sedentary behaviour and cardiometabolic risk in older adults. Prev Med. 2012;54:82-87. doi:10.1016/j.ypmed.2011.10.009

31. Fasbender U, Deller J, Wang M, et al. Deciding whether to work after retirement: the role of the psychological experience of aging. $J$ Voc Behav. 2014;8:215-224. doi:10.1016/j.jvb.2014.01.006

32. Office for National Statistics. Commuting and Personal Well Being. London: Office of National Statistics; 2014.

33. Hamer M, Stamatakis E. U-shaped association between body-mass index and psychological distress in a population of 114,218 British adults. Mayo Clin Proc. 2017;92:1865-1866. doi:10.1016/j.mayocp. 2017.09.014

34. Azevedo LB, Ling J, Soos I, et al. The effectiveness of sedentary behaviour interventions for reducing body mass index in children and adolescents: systematic review and meta-analysis. Obes Rev. 2016;17:623-635. doi:10.1111/obr.12414

35. Serreti A, Fabbri C. Shared genetics among major psychiatric disorders. Lancet. 2013;381:1339-1341. doi:10.1016/S0140-6736(13)60223-8
Psychology Research and Behavior Management

\section{Publish your work in this journal}

Psychology Research and Behavior Management is an international, peer-reviewed, open access journal focusing on the science of psychology and its application in behavior management to develop improved outcomes in the clinical, educational, sports and business arenas. Specific topics covered in the journal include: Neuroscience, memory and decision making; Behavior modification and management; Clinical applications; Business and sports performance management; Social and developmental studies; Animal studies. The manuscript management system is completely online and includes a very quick and fair peer-review system, which is all easy to use. Visit http://www. dovepress.com/testimonials.php to read real quotes from published authors. 